\title{
Prévalence de la lipodystrophie et facteurs associés chez les personnes adultes vivant avec le VIH sous antirétroviral suivies à l'hôpital Béthesda de Cotonou en 2014.
}

\section{Prevalence of lipodystrophy and associated factors in adults living with HIV on antiretroviral followed Bethesda Hospital in Cotonou in 2014.}

\author{
ALASSANI A ${ }^{1}$, DOVONOU AC ${ }^{1}$, ATTINSOUNON AC ${ }^{1}$, GNINKOUN J ${ }^{2}$, WANVOEGBE A ${ }^{3}$, CODJO L ${ }^{1}$, \\ ZANNOU DM ${ }^{2}$, DJROLO $\mathrm{F}^{2}$, HOUNGBE $\mathrm{F}^{2}$.
}

1 Centre Hospitalier Universitaire Départemental du Borgou-Alibori, Parakou, Bénin

2 Centre National Hospitalier Universitaire Hubert Koutoucou Maga, Cotonou, Bénin

3 Centre Hospitalier Universitaire Départemental de l'Ouémé-Plateau, Porto-Novo, Bénin

Auteur correspondant : ALASSANI Adébayo, médecin interniste au Centre Hospitalier Universitaire

Départemental du Borgou-Alibori, Service de Médecine Interne, mail : alsaco2007@yahoo.fr

\section{Résumé}

Objectif : l'objectif de cette étude est de déterminer la prévalence et les facteurs associés à la lipodystrophie chez les personnes vivant avec le VIH sous antirétroviral suivies à l'hôpital Béthesda de Cotonou.

Méthodes : Il s'est agi d'une étude transversale, descriptive et analytique. La population d'étude est constituée par les personnes vivant avec le VIH sous antirétroviral, suivies à l'hôpital Béthesda de Cotonou. La lipodystrophie a été définie par la présence de signes de lipoatrophie et/ou de lipohypertrophie objectivés par les patients et confirmés par l'examen clinique.

Résultats : Au total 305 patients ont été inclus dans l'étude, dont 214 (79\%) de sexe féminin. L'âge moyen des

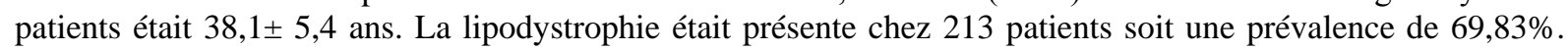
Les facteurs associés à la lipodystrophie étaient le sexe féminin, l'âge de 40 ans et plus, la sédentarité, la consommation du tabac, l'apport en énergie anormal, la consommation du tabac, la consommation insuffisante en fruits et légumes, une durée de traitement antirétroviral de 1 an ou plus, un taux de CD4 inférieur à 200 cellules $/ \mathrm{mm}^{3}$ au dépistage, l'absence d'appui alimentaire et de conseils nutritionnels, un IMC anormal et un tour de taille élevé.

Conclusion : La lipodystrophie est fréquente chez les patients infectés par le VIH.

Mots clés: Lipodystrophie, VIH, Cotonou, Bénin

\section{Summary}

Objectives: The objective of this study was to determine the prevalence of lipodystrophy and associated factors in people living with HIV on antiretroviral followed in Bethesda Hospital, Cotonou.

Methodology: cross-sectional study, descriptive and analytical. The study population consists of people living with HIV on antiretroviral followed in Bethesda Hospital in Cotonou. Lipodystrophy was defined by the presence of signs of lipoatrophy and/or lipohypertrophy objectified by patients and confirmed by clinical examination.

Results: In total 305 patients were included in the study, 214 (79\%) were female. The mean age of patients was $38.1 \pm 5.4$ years. Lipodystrophy was present in 213 patients a prevalence of $69.83 \%$. Factors associated with lipodystrophy were female gender, age 40 and older, physical inactivity, tobacco use, abnormal energy intake, consumption of tobacco, insufficient consumption of fruits and vegetables, an antiretroviral treatment duration of 1 year or more, a CD4 count below 200 cells $/ \mathrm{mm}^{3}$ at screening, lack of food support and nutritional advice, abnormal BMI and high waist measurement.

Conclusion: Lipodystrophy is common in patients with HIV infection.

Key worlds: Lipodystrophy, PVVIH, Cotonou, Benin

\section{Introduction}

Depuis l'identification du premier cas d'infection VIH en 1981, on a assisté à une augmentation progressive du nombre de personnes atteintes. L'infection VIH a posé il y a quelques années un sérieux problème de santé de part la morbidité et la mortalité qu'elle engendre [1]. De nos jours, avec le traitement antirétroviral très actif, on a assisté à une diminution considérable de l'incidence des affections opportunistes et à la réduction de la mortalité liées au VIH [2]. L'infection au VIH est devenue aujourd'hui une maladie chronique [3]. Les sujets infectés ont de plus en plus une augmentation de l'espérance de vie et une amélioration de la qualité de vie [4]. Cependant, malgré les effets bénéfiques du 
traitement antirétroviral, on assiste à l'apparition des anomalies métaboliques. Parmi ces anomalies, la lipodystrophie fait partie des plus fréquentes. Elle est caractérisée par une redistribution de la graisse corporelle avec accumulation tronculaire de la graisse et une perte de graisse au niveau périphérique [5]. La pathogénie de la lipodystrophie chez les personnes vivant avec le VIH (PVVIH) sous antirétroviral n'est pas encore clairement définie [6]. Plusieurs facteurs associés à la lipodystrophie ont été décrits. Parmi ces facteurs on cite les antirétroviraux tels que les inhibiteurs nucléosidiques de la transcriptase inverse et les antiprotéases, le VIH, les facteurs génétiques, le mode de vie des patients, les facteurs environnementaux [7,8]. Les conséquences de la lipodystrophie sont nombreuses. Elle est source de conséquences esthétiques, de stigmatisation, d'abandon du traitement, de perte de l'emploi, de l'altération de la qualité de vie et parfois de violences physiques [9, 10, 11]. Elle est également associée au risque de développer l'insulinorésistance, le diabète de type 2, les dyslipidémies et les maladies cardiovasculaires [12, 13, 14]. La présente étude a pour but de déterminer la prévalence des lipodystrophies et d'identifier les facteurs favorisants dans le contexte béninois pour améliorer la prise en charge des patients sous antirétroviraux.

\section{Patients et méthode}

Il s'est agi d'une étude transversale, descriptive et analytique. La population d'étude est constituée des patients adultes vivant avec le VIH sous traitement antirétroviral suivis à l'hôpital Béthesda de Cotonou en 2014. Les patients hospitalisés, convalescents ou incapables de répondre aux questions ont été exclus de l'étude. Il en est de même des patients ayant une variation pondérale de plus ou moins $10 \%$ du poids corporel par rapport au poids antérieur il y a 6 mois ou ayant des œdèmes des membres inférieurs. La lipodystrophie est définie par la présence des signes suivants signalés par le patient et objectivés à l'examen. Il s'agit de la présence de lipohypertrophie définie par la présence d'au moins un des signes suivants: accumulation de la graisse au niveau du cou ou du dos, augmentation du volume des seins ou de l'abdomen; de lipoatrophie définie par la présence d'au moins un des signes suivants : fonte musculaire au niveau des membres, des fesses ou au niveau de la face (région temporale), saillie anormale des veines superficielles au niveau des membres, vieillissement de la peau. La présence de signes de lipoatrophie et de lipohypertrophie définit le syndrome de lipodystrophie mixte. L'évaluation des apports en nutriments a été faite par le journal alimentaire chez les patients. Les besoins énergétiques ont été calculés par la formule de Harris et Bénédict. Un apport énergétique inférieur à plus de $500 \mathrm{kcal}$ des besoins énergétiques est considéré comme bas et lorsqu'il est supérieur de plus de $500 \mathrm{kcal}$, il est élevé. Les apports énergétiques bas et élevé sont considérés comme anormaux. L'apport lipidique est considéré comme élevé lorsqu'il est supérieur à $30 \%$ de l'apport énergétique journalier. Une portion de fruit équivaut à la taille d'une orange, celle des légumes équivaut à un volume de $250 \mathrm{~mL}$. Un apport journalier en fruits et légumes inférieur à 5 portions est considéré comme insuffisant. Les patients ayant manqué au moins $5 \%$ la prise des antirétroviraux ou du cotrimoxazole au cours des 7 derniers jours sont considérés comme mauvais observants au traitement. L'indice de masse corporelle (IMC) est calculé en faisant le rapport du poids en $\mathrm{kg}$ par la taille au carré en $\mathrm{m}^{2}$. La dénutrition est définie par un IMC inférieur à $18,5 \mathrm{~kg} / \mathrm{m}^{2}$, le poids normal pour un IMC supérieur ou égal à 18,5 et inférieur à $25 \mathrm{~kg} / \mathrm{m}^{2}$, le surpoids pour un IMC supérieur ou égal à 25 et inférieur à $30 \mathrm{~kg} / \mathrm{m}^{2}$ et l'obésité pour un IMC supérieur ou égal à $30 \mathrm{~kg} / \mathrm{m}^{2}$. Les patients dénutris, en surpoids ou obèses sont considérés comme ayant un indice de masse corporelle anormal. Le tour de taille est élevé lorsqu'il est supérieur ou égal à $80 \mathrm{~cm}$ chez la femme ou supérieur ou égal à $94 \mathrm{~cm}$ chez l'homme. L'analyse des données a été faite par le logiciel Epi DATA 3.1. L'association entre la variable dépendante et les variables indépendantes était faite par le test de $\mathrm{Chi}^{2}$ de Person pour les effectifs supérieurs ou égaux à 5 . Le test de $\mathrm{Chi}^{2}$ corrigé de Yates a été utilisé pour les effectifs inférieurs à 5 . Une p-value $<0,05$ a été considérée comme statistiquement significative.

\section{Résultats}

Au total 305 patients ont été inclus. On a noté une prédominance féminine (79\%). L'âge moyen était de $38,1 \pm 5,4$ ans avec une prédominance des sujets âgés de moins 40 ans (56\%). La majorité des patients $(72,46 \%)$ était sédentaire, ne consommait pas d'alcool $(77,70 \%)$ et non tabagique $(96,73 \%)$. Seulement $26(8,52 \%)$ et $19(6,23 \%)$ patients avaient respectivement un apport énergétique normal et un apport lipidique élevé. La plupart des patients $(91,80 \%)$ avaient un apport journalier en fruits et légumes insuffisant. Vingt sept patients $(8,85 \%)$ prenaient un traitement antirétroviral depuis moins d'un an. Le taux de CD4 au dépistage était inférieur à 200 cellules $/ \mathrm{mm}^{3}$ chez 192 patients $(91 \%)$. Près du tiers des patients $(30,16 \%)$ avaient un appui alimentaire et 4 patients sur $40(40,98 \%)$ ont reçu des conseils alimentaires. Le schéma thérapeutique dominant associait deux inhibiteurs nucléosidiques de la transcriptase inverse (INTI) et un inhibiteur non nucléosidique de la transcriptase inverse (INNTI) dans 98,69\% des cas (Tableau I). Les INTI utilisés étaient lamivudine chez tous les patients, zidovudine chez 
290 patients $(95,08 \%)$ et ténofovir chez 15 patients $(4,92 \%)$. Les INNTI utilisés sont l'éfavirenz chez 273 patients $(89,5 \%)$ et la névirapine chez 32 patients (10,5\%). La lipodystrophie était observée chez 213 patients soit une prévalence de 69,83\% avec une prédominance de la lipohypertrophie (155 patients). La dénutrition était observée chez 24 patients $(7,83 \%)$. Le tour de taille était élevé chez 177 patients $(58,04 \%)$. Tableau II. Les facteurs associés à la lipodystrophie étaient le sexe féminin, l'âge de 40 ans et plus, la sédentarité, la consommation du tabac, l'apport en énergie anormal, la consommation $\mathrm{du}$ tabac, la consommation insuffisance en fruits et légumes, une durée de traitement antirétroviral de 1 an ou plus, un taux de CD4 inférieur à 200 cellules $/ \mathrm{mm}^{3}$ au dépistage, l'absence d'appui alimentaire et de conseils nutritionnels, un IMC anormal et un tour de taille élevé (Tableaux III et IV).

\section{Discussion}

La présente étude a permis de déterminer la prévalence de la lipodystrophie chez les PVVIH sous traitement antirétroviral, ainsi que les facteurs associés. Les résultats de cette étude montrent une prévalence de lipodystrophie de l'ordre de $70 \%$ chez les PVVIH. Plusieurs auteurs ont confirmé cette prévalence élevée. C'est le cas des études de Finkelstein [2] et Estrada [15] qui signalent respectivement une prévalence de lipodystrophie de $63 \%$ et de $67,3 \%$. Il faut remarquer la jeunesse de la population d'étude et la prédominance des femmes. Cette jeunesse des patients infectés par le VIH est aussi soulignée dans les études de Trevisol et al [6] et de Tsuda et al [11] avec des moyennes d'âge respectives de $39,1 \pm 10,1$ ans et de $43,08 \pm 2,81$ ans. La prédominance féminine est rapportée par Berhane et al [4]. La lipodystrophie a été associée aux facteurs de risque suivants: le sexe féminin, l'âge de 40 ans et plus, la sédentarité, la consommation du tabac, l'apport en énergie anormal, la consommation insuffisance en fruits et légumes, une durée de traitement antirétroviral de 1 an ou plus, un taux de CD4 inférieur à 200 cellules $/ \mathrm{mm}^{3}$ au dépistage, l'absence d'appui alimentaire et de conseils nutritionnels, un IMC anormal et un tour de taille élevé. Ces différents facteurs de risque sont retrouvés dans d'autres études. L'association entre sexe féminin et lipodystrophie est rapportée par Berhane [4] et Ali [13]. Dans les études de Segatto [9] et Justina [7], l'âge élevé est associé à la lipodystrophie. Segatto et al [9] a confirmé l'association entre la sédentarité et la lipodystrophie. L'activité physique par le biais de la dépense énergétique peut réduire les réserves en graisse de l'organisme et contribue donc à la réduction de l'obésité abdominale et de la lipohypertrophie abdominale. La consommation du tabac est associée à la lipoatrophie selon Zannou [16]. L'efficacité d'un régime alimentaire incluant un apport énergétique normal et la consommation en quantité suffisante de fruits et légumes dans la lutte contre la lipodystrophie a été retrouvée dans d'autres études [2, 17]. L'étude de Papi [18] a confirmé l'importance du régime et a montré que l'excès de consommation en lipide et l'apport insuffisant en fruits et légumes sont associés à la lipodystrophie. Il est donc important de donner des conseils nutritionnels aux patients avec si besoin un soutien alimentaire. La durée du traitement comme un facteur de risque de la lipodystrophie a été confirmée par Bhutia [5] et Yonaba [14]. Les sujets ayant un statut immunitaire à moins de $200 \mathrm{CD}_{4}$ $/ \mathrm{mm}^{3}$ à l'admission étaient les plus exposés dans la présente étude. Dans l'étude de Berhane [14] le stade IV OMS est associé à la lipodystrophie. Pour Trevisol [6], le taux de lymphocytes $\mathrm{CD}_{4}$ bas et une charge virale détectable sont associés à la lipodystrophie. Toutes les études démontrent que la lipodystrophie est multifactorielle. Les facteurs de risque non modifiables de la lipodystrophie sont le sexe, l'âge et la durée du traitement. Par contre, une bonne hygiène de vie et un dépistage précoce de l'infection au VIH doivent permettre de réduire la prévalence de la lipodystrophie.

\section{Conclusion}

La lipodystrophie est très fréquente chez les PVVIH.

\section{Références}

1 Verolet CM, Delhumeau-Cartier C, Sartori M, Toma S, Zawadynski S, Becker M. Lipodystrophy among HIV-infected patients: a cross-sectional study on impact

on quality of life and mental health disorders. AIDS Res Ther 2015; 12 (1) :21-30

2 Finkelstein JL, Gala P, Rochford R, Glesby MJ, Mehta S. HIV/AIDS and lipodystrophy: Implications for clinical management in resourcelimited settings. Journal of the International AIDS Society 2015;18(1):1-20

3 Justina L, Trevisol D, Trevisol F. Prevalence of HIV-Associated Lipodystrophy in Brazil: A Systematic Review of the Literature. AIDS Clin Res 2014; 5(9): 1-6

4 Berhane T, Yami A, Alemseged F, Yemane T, Hamza L, Kassim M. Prevalence of lipodystrophy and metabolic syndrome among HIV positive individuals

on Highly Active Anti-Retroviral treatment in Jimma, South West Ethiopia. Pan African Medical Journal 2013; 13(1): 43-56

5 Bhutia E, Hemal A, Yadav TP, Ramesh KL. Lipodystrophy syndrome among HIV infected children on highly active antiretroviral therapy in northern India. African Health Sciences 2014; 14(2):408-13

6 Trevisol FS, Alencastro PR, Ribeiro P, Wollf FH, Ikeda M, Barcellos N. Association of 
Physical Activity with Lipodystrophy Syndrome in HIVInfected Patients. J AIDS Clinic Res 2012; 3(1):177-82

7 Justina L, Luiz M, Maurici R, Trevisol F. Prevalence and factors associated with lipodystrophy in AIDS patients. Revista da Sociedade Brasileira de Medicina Tropical 2014; 47(1):30-7

8 Lana L, Junqueira D, Perini E, de Pádua C. Lipodystrophy among patients with

HIV infection on antiretroviral therapy: a systematic review protocol. BMJ Open 2014; 4(3): $1-5$

9 Segatto AF, Junior IF, dos Santos VR, Cristina K, Alves P, Barbosa DA. Lipodystrophy in HIV/AIDS patients with different levels of physical activity while on antiretroviral therapy. Revista da Sociedade Brasileira de Medicina Tropical 2011; 44(4):420-4

10 Innes S, Cotton MF, Haubrich R, Conradie M, van Niekerk M, Edson C. High prevalence of lipoatrophy in pre-pubertal South African children on antiretroviral therapy: a cross-sectional study. BMC Pediatrics 2012; 12(1):183-90

11 Tsuda LC, da Silva M, Machado A, Fernandes A. Body changes: antiretroviral therapy and lipodystrophy syndrome in people living with HIV/aids. Rev. Latino-Am. Enfermagem 2012; 20(5):847-53

12 Alves M, Brites C, Sprinz E. HIVassociated lipodystrophy: a review from a Brazilian perspective. Therapeutics and Clinical Risk Management 2014;10(1):559-66.
13 Ali M, Magee M, Dave J, Ofotokun I, Tungsiripat, Jones T. MDHIV and Metabolic, Body, and Bone Disorders: What We Know From Low- and Middle-Income Countries. J Acquir Immune Defic Syndr 2014; 67(1) :27-39

14 Yonaba C, Ouedraogo A, Ouédraogo S, Ouattara B, Kalmogho A, Koueta F. Lipodystrophy among Children Infected with Human Immunodeficiency Virus and on Antiretroviral Treatment in Ouagadougou. Open Journal of Pediatrics 2015; 5(3):199-206

15 Estrada V, Martinez-Larrad M, GonzalezSanchez J, de Villar N, Zabena C, Fernandez C. Lipodystrophy and metabolic syndrome in HIVinfected patients treated

with antiretroviral therapy. Metabolism Clinical and Experimental 2006; 55(7): 940- 945

16 Zannou DM, Denoeud L, Lacombe K, Amoussou-Guenou D, Bashi J, Akakpo J. Incidence of lipodystrophy and metabolic disorders in patients starting non nucleoside reverse transcriptase inhibitors in Benin. Antiviral Therapy 2009; 14(3): 371-380

17 Singhania R, Kotler D. Lipodystrophy in HIV patients: its challenges

and management approaches. HIV/AIDS Research and Palliative Care 2011; 3(1): 135-143

18 Papi L, Menezes A, Rocha H, Abreu T, de Oliveira R, Frota A. Prevalence of lipodystrophy and risk factors for dyslipidemia in HIV-infected children in Brazil. Braz J Infect Dis 2014;1 8(4):394-9 
Tableau I : Caractéristiques générales des personnes adultes vivant avec le VIH sous antirétroviral suivis à l'hôpital Béthesda de Cotonou en 2014

\begin{tabular}{|c|c|c|c|}
\hline Variables & $\mathrm{n}(\%)$ & Variables & $\mathrm{n}(\%)$ \\
\hline \multicolumn{4}{|c|}{ Taille de la population d'étude : 305} \\
\hline \multicolumn{2}{|l|}{ Sexe } & \multicolumn{2}{|l|}{ Age } \\
\hline Femmes & 241(79) & Moins de 40 ans & $171(56)$ \\
\hline Hommes & $64(21)$ & 40 ans et plus & $134(44)$ \\
\hline \multicolumn{2}{|c|}{ Niveau d'activité physique } & \multicolumn{2}{|c|}{ Consommation d'alcool } \\
\hline Sédentaire & $221(72,46)$ & Oui & $68(22,30)$ \\
\hline Actif & $84(27,54)$ & Non & $237(77,70)$ \\
\hline \multicolumn{2}{|c|}{ Consommation de tabac } & \multicolumn{2}{|c|}{ Apport énergétique } \\
\hline Oui & $10(3,27)$ & Bas & $153(50,16)$ \\
\hline \multirow[t]{2}{*}{ Non } & $295(96,73)$ & Normal & $26(8,52)$ \\
\hline & & Elevé & $126(41,32)$ \\
\hline \multicolumn{2}{|c|}{ Apport en lipide } & \multicolumn{2}{|c|}{ Consommation de fruits et légumes } \\
\hline Normal & $286(93,77)$ & Insuffisant & $280(91,80)$ \\
\hline Elevé & $19(6,23)$ & Normal & $25(8,20)$ \\
\hline \multicolumn{2}{|c|}{ Durée de traitement antirétroviral } & \multicolumn{2}{|c|}{ Observance du traitement antirétroviral } \\
\hline Moins 1 an & $27(8,85)$ & Bonne & $194(63,61)$ \\
\hline 1 an ou plus & $278(91,15)$ & Mauvaise & $111(36,39)$ \\
\hline \multicolumn{2}{|c|}{ Observance du cotrimoxazole } & \multicolumn{2}{|c|}{ Taux de CD4 à l'admission $(n=211)$} \\
\hline Bonne & $122(40)$ & Moins de 200 & 192(91) \\
\hline Mauvaise & $183(60)$ & 200 ou plus & $19(9)$ \\
\hline \multicolumn{2}{|c|}{ Appui alimentaire } & \multicolumn{2}{|c|}{ Conseils alimentaires } \\
\hline Oui & $92(30,16)$ & Oui & $125(40,98)$ \\
\hline Non & $213(69,84)$ & Non & $180(59,02)$ \\
\hline \multicolumn{4}{|c|}{ Type de traitement ARV } \\
\hline 2INTI+1INNTI & $301(98,69)$ & & \\
\hline 2INTI+IP & $4(1,31)$ & & \\
\hline
\end{tabular}


Tableau II : Répartition des personnes adultes vivant avec le VIH sous antirétroviral suivis à l'hôpital Béthesda de Cotonou en 2014 en fonction de la présence de lipodystrophie, du type de lipodystrophie, de l'indice de masse corporelle et du tour de taille

\begin{tabular}{lc}
\hline Variables & $\mathbf{n}(\boldsymbol{\%})$ \\
\hline Taille de la population d'étude $: \mathbf{3 0 5}$ & \\
Lipodystrophie & $\mathbf{2 1 3}(\mathbf{6 9 , 8 3})$ \\
Lipohypertrophie & $155(50,82)$ \\
Lipoatrophie & $54(17,70)$ \\
Syndrome mixte & $4(1,31)$ \\
Indice de masse corporelle & \\
Dénutrition & $24(7,87)$ \\
Normal & $185(60,66)$ \\
Surpoids & $59(19,34)$ \\
Obésité & $37(12,13)$ \\
Tour de taille & \\
Elevé & $177(58,04)$ \\
Normal & $128(41,96)$ \\
\hline
\end{tabular}


Tableau III : Facteurs associés à la lipodystrophie des personnes adultes vivant avec le VIH sous antirétroviral suivis à l'hôpital Béthesda de Cotonou en 2014 (Analyse univariée)

\begin{tabular}{|c|c|c|c|}
\hline Variables & Lipodystrophie n(\%) & Pas de lipodystrophie n(\%) & p value \\
\hline \multicolumn{4}{|c|}{ - } \\
\hline Femmes & $180(74,68 \%)$ & $61(25,32 \%)$ & 0,00034 \\
\hline Hommes & $33(51,56 \%)$ & $31(48,44 \%)$ & \\
\hline \multicolumn{4}{|l|}{ Age } \\
\hline$<40$ ans & $109(63,74 \%)$ & $62(36,26 \%)$ & 0,0088 \\
\hline 40 ans et plus & $104(77,61 \%)$ & $30(22,39 \%)$ & \\
\hline \multicolumn{4}{|c|}{ Niveau d'activité physique } \\
\hline Sédentaire & $163(73,76 \%)$ & $58(26,24 \%)$ & $\mathbf{0 , 0 1 5 5}$ \\
\hline Actif & $50(59,52 \%)$ & $34(40,48 \%)$ & \\
\hline \multicolumn{4}{|c|}{ Consommation de l'alcool } \\
\hline Oui & $51(75 \%)$ & $17(25 \%)$ & 0,2925 \\
\hline Non & $162(68,35 \%)$ & $75(31,65 \%)$ & \\
\hline \multicolumn{4}{|c|}{ Consommation de tabac } \\
\hline Oui & $10(100 \%)$ & $0(0 \%)$ & $\mathbf{0 , 0 3 4 5 8}$ \\
\hline Non & $203(68,81 \%)$ & $92(31,19 \%)$ & \\
\hline \multicolumn{4}{|c|}{ Apport énergétique } \\
\hline Normal & $3(11,54 \%)$ & $23(88,46 \%)$ & 0,00000 \\
\hline Anormal & $200(71,68 \%)$ & $79(28,32 \%)$ & \\
\hline \multicolumn{4}{|c|}{ Apport en lipide } \\
\hline Normal & $197(68,88 \%)$ & $89(31,12 \%)$ & $\mathbf{0 , 1 5 8 6}$ \\
\hline Elevé & $16(84,21 \%)$ & $3(15,79 \%)$ & \\
\hline \multicolumn{4}{|c|}{ Consommation de fruits et légumes } \\
\hline Insuffisant & $208(74,29 \%)$ & $72(25,71 \%)$ & $\mathbf{0 , 0 0 0 0 0}$ \\
\hline Normal & $5(20 \%)$ & $20(80 \%)$ & \\
\hline \multicolumn{4}{|c|}{ Durée du traitement antirétroviral } \\
\hline Moins 1 an & $5(18,5 \%)$ & $22(81,5 \%)$ & $\mathbf{0 , 0 0 0 0 0}$ \\
\hline 1 an ou plus & $208(74,29 \%)$ & $70(25,71 \%)$ & \\
\hline
\end{tabular}


Tableau IV : Facteurs associés à la lipodystrophie des personnes adultes vivant avec le VIH sous antirétroviral suivis à l'hôpital Bétheda de Cotonou en 2014 (Analyse univariée suite et fin)

\begin{tabular}{|c|c|c|c|}
\hline Variables & Lipodystrophie & Pas de lipodystrophie & p value \\
\hline \multicolumn{4}{|c|}{ Observance des antirétroviraux } \\
\hline Oui & $130(67,01)$ & $64(32,99 \%)$ & 0,155 \\
\hline Non & $83(74,77)$ & $28(25,23 \%)$ & \\
\hline \multicolumn{4}{|c|}{ Observance du cotrimoxazole } \\
\hline Oui & $90(73,77 \%)$ & $32(26,23 \%)$ & 0,2215 \\
\hline Non & $123(67,21 \%)$ & $60(32,79 \%)$ & \\
\hline \multicolumn{4}{|c|}{ Taux de CD4 au dépistage } \\
\hline Moins de 200 & $150(78,13 \%)$ & $42(21,87 \%)$ & 0,00000 \\
\hline 200 ou plus & $3(15,79 \%)$ & $16(84,21 \%)$ & \\
\hline \multicolumn{4}{|c|}{ Appui alimentaire } \\
\hline Non & $159(74,65 \%)$ & $54(25,35 \%)$ & \\
\hline \multicolumn{4}{|c|}{ Conseils alimentaires } \\
\hline Oui & $45(36 \%)$ & $80(64 \%)$ & 0,00000 \\
\hline Non & $168(93,33 \%)$ & $11(6,67 \%)$ & \\
\hline \multicolumn{4}{|c|}{ Type de traitement antirétroviral } \\
\hline 2INTI+1INNTI & $210(69,77 \%)$ & $91(30,23 \%)$ & $\mathbf{0 , 8 2 0 8}$ \\
\hline 2INTI+IP & $3(75 \%)$ & $1(25 \%)$ & \\
\hline \multicolumn{4}{|c|}{ Indice de masse corporelle } \\
\hline Normal & $100(54,05 \%)$ & $85(45,95 \%)$ & 0,00000 \\
\hline Anormal & $113(94,17 \%)$ & $7(5,83 \%)$ & \\
\hline \multicolumn{4}{|l|}{ Tour de taille } \\
\hline Elevé & $175(98,87 \%)$ & $2(1,13 \%)$ & 0,0176 \\
\hline Normal & $38(29,69 \%)$ & $90(70,31 \%)$ & \\
\hline
\end{tabular}


Tableau V : Regression logistique des facteurs associés à la lipodystrophie des personnes adultes vivant avec le VIH sous antirétroviral suivis à l'hôpital Bétheda de Cotonou en 2014

\begin{tabular}{|c|c|c|}
\hline Variables & Odds Ratio (IC à95\%) & p-value \\
\hline \multicolumn{3}{|l|}{ Sexe } \\
\hline Femmes & 1 & 0,0000 \\
\hline Hommes & $0,6(0,45 ; 0,89)$ & \\
\hline \multicolumn{3}{|l|}{ Age } \\
\hline$<40$ ans & 1 & 0,0000 \\
\hline 40 ans et plus & $1,85(1,31 ; 2,39)$ & \\
\hline \multicolumn{3}{|c|}{ Niveau d'activité physique } \\
\hline Sédentaire & 1 & 0,0056 \\
\hline Actif & $2,06(1,44 ; 3,02)$ & \\
\hline \multicolumn{3}{|c|}{ Consommation de tabac } \\
\hline Oui & 1 & 0,345 \\
\hline Non & $0,8(0,7 ; 1,56)$ & \\
\hline \multicolumn{3}{|c|}{ Apport énergétique } \\
\hline Normal & 1 & 0,0000 \\
\hline Anormal & $3,22(2,38 ; 7,89)$ & \\
\hline \multicolumn{2}{|c|}{ Consommation en fruits et légumes } & 0,0000 \\
\hline Insuffisant & $2,89(1,99 ; 4,33)$ & \\
\hline \multicolumn{2}{|c|}{ Durée du traitement antirétroviral } & 0,0037 \\
\hline 1 an ou plus & $2,22(1,77 ; 4,02)$ & \\
\hline \multicolumn{3}{|c|}{ Taux de CD4 au dépistage } \\
\hline 200 ou plus & $0,54(0,33 ; 0,76)$ & \\
\hline \multicolumn{3}{|c|}{ Appui alimentaire } \\
\hline Oui & 1 & 0,572 \\
\hline Non & $1,31(0,79 ; 2,03)$ & \\
\hline \multicolumn{3}{|c|}{ Conseils alimentaires } \\
\hline Oui & 1 & 0,143 \\
\hline Non & $2,03(0,89 ; 3,13)$ & \\
\hline \multicolumn{3}{|c|}{ Indice de masse corporelle } \\
\hline Normal & 1 & 0,001 \\
\hline Anormal & $3,49(2,45 ; 5,17)$ & \\
\hline \multicolumn{3}{|l|}{ Tour de taille } \\
\hline Elevé & 1 & 0,046 \\
\hline Normal & $2,89(1,58 ; 4,78)$ & \\
\hline
\end{tabular}

